\title{
The Design and Development of a Multi-Lingual Braille System Output Device with Audio Enhancement
}

\author{
Michel J. Owayjan, Taimour Z. Wehbe, Elie Y. Bou Daher, Omran A. Ayoub
}

Department of Computer and Communications Engineering, American University of Science \& Technology, Beirut, Lebanon. Email: mowayjan@aust.edu.lb, taimourwehbe@live.com, elie-bou-daher@hotmail.com,omran.ayoub@hotmail.com

Received March $10^{\text {th }}, 2013$; revised April 11 ${ }^{\text {th }}, 2013$; accepted April $19^{\text {th }}, 2013$

Copyright (c) 2013 Michel J. Owayjan et al. This is an open access article distributed under the Creative Commons Attribution License, which permits unrestricted use, distribution, and reproduction in any medium, provided the original work is properly cited.

\begin{abstract}
Visually impaired people face difficulties in interacting and gaining full advantage of computers. Recently, and with the fast evolution in technology, researchers proposed to give the blinds the ability to take advantage of these advancements. Accordingly, designers and engineers started working on projects that relate input and output devices to the computers in order for the blind individual to have full control of the hi-tech machines. However, investments in these kinds of hardware presented complexity in the design, in addition to the high cost imposed by the devices used. The project's objective is to design and develop a Multi-Lingual Braille System Output Device for the visually impaired individuals that enable them to access and read texts from a computer. The device acquires both English or Arabic texts and displays them using controlled piezoelectric Braille cells. It also has the ability to produce a sound that matches the displayed characters. The control of the cells is done via Programmable Interface Controller (PIC) microcontroller. The interaction between the device and the computer is done through the Universal Serial Bus (USB). C\# is the language used to write the program responsible for controlling this interaction. The continuous development of such devices is essential for the visually impaired to keep up with the technological advancements. The main advantages of this system are its low cost, the added multi-lingual, and the audio features. In addition, many features can be added so as to satisfy the users' needs, such as adding new languages and Grade 2 Braille System.
\end{abstract}

Keywords: Assistive Technologies; Braille Devices; Computer Peripheral Parts; Low-Vision Aiding Systems; Rehabilitation Engineering

\section{Introduction}

The idea behind the Universal Braille System started in 1821 by Louis Braille [1-3]. In 1829, Braille developed the Braille System. Since then, the Braille system has become the most adopted system for written communication for blind and visually impaired individuals [4].

The Braille system is a method which translates letters to a combination of dots in order to help individuals with sight defect read documents. This system consists of three grades and two methods of representing the characters. The grades are introduced in the Braille system to make the reading of documents more dynamic and faster. The two methods are either using a six-dot cell or an eight-dot cell. In addition to the grades, there are four Braille codes $[5,6]$. The codes are the Literary Braille Code, the Nemeth Braille Code, the Computer Braille Code, and the Music Braille Code [7].

To this date, there are no other practical means that re- places this system. Using Braille, blind individuals can read a character by touch. The individual can read a Braille character by rolling a finger over its six-dot or its eight-dot representation. The combinations of these dots are specifically formed by raising the pins to represent a certain letter or symbol. A Braille reader can learn how to read Braille characters by first learning the position of the dots on the cell, and then by memorizing the representations of all the letters, numbers and characters. In Grade 2 Braille, the user needs to learn additional contractions [5].

The fast progress in technology urged scientists and engineers to design and work on projects that help the visually impaired people interact with current advancements. Assistive technologies were introduced to help people with disabilities in managing their daily chores. However, such introduced devices imposed high cost; thus, hindering their availability in the hands of disabled people. 
The Multi-Lingual Braille System Output Device with Audio Enhancement can significantly help visually impaired individuals in managing their daily computerrelated activities; it particularly helps visually impaired students at schools and universities. Moreover, an audio system is integrated to help the visually impaired individuals to differentiate between similar characters. This system can also be used in institutes for the blind to simplify the learning process of the Braille system. Furthermore, the integration of the Arabic language adds to the benefits of this device; especially, in Lebanon and the Arab World.

\section{Literature Review}

In the past two decades, several Braille System Output Devices were developed to help the blind and visually impaired individuals interact with computers. In the late 1990's, a device called Braille Lite was developed by Blazie Engineering (Windermere House, Kendal Avenue, London, England) [8,9]. It has the ability to represent up to 18 characters and is refreshable; characteristics that help visually impaired individuals to skim a text document more easily. However, its price made it difficult for all visually-impaired and blind individuals to acquire it [10].

In order to give blind persons the ability to read characters easily, Telesensory (Bukit Batok Crescent, Prestige Centre, Singapore) developed a device called Optacon. Optacon is a device that prints the regular shape of the letters, and enables the readers to feel the letter and thus read it. This was done by using vibrating metal rods that are moved over a printed page, converting the image of the letter into a tactile form [11]. The main drawback of the Optacon is that it was not very practical, and it would take a visually-impaired person a very long time to skim a text document [12].

In 2001, the Palm Braille was developed by Scott Stoffel. It is a device which connects to the parallel port of the computer and has the ability to read the characters from a file in order to output them. The users of the device can sense the six pins on the device and know which letter they are reading. The problem of the Palm Braille is that it was only able to read one character at a time [11]. To make reading easier and the time spent by users more efficient, Benetech ${ }^{\circledR}$ (California Avenue, CA, USA) translated more than 7000 books into Braille language and made them available online. This action helps the blind or visually-impaired individuals to print out a book using Braille printers and read it on papers easily, but it does not help others who wish to read books that are not available online [13].

Also in 2001, the Alva Refreshable Braille and the Alva 570 Satellite Pro were introduced by Optelec (Breslau, LT Barendrecht, Netherlands) [14]. The Alva
Refreshable Braille 8-dots cells refresh and change according to the part of the screen that has the computer's attention; whereas, the Alva 570 Satellite Pro was the first designed desktop model that gives the user the ability to read a whole line of 80 cells each [14]. On one hand, these two developments solved the problems that all previous Braille display systems encountered, but on the other hand, each of the mentioned developments would cost a user more than five thousand US dollars, which is considered to be very expensive [15].

The problem with the old versions of the Braille output devices is that they are time consuming or very expensive or even both. The solution that solved the first problem is the invention of the Dynamic and Refreshable Braille Output Device. The Refreshable Braille display uses 40 or 80 cells lines and outputs one line at a time [15]. The device connects to the computer using a serial cable and displays the characters with a ribbon of hard rubber. The device has many advantages as it is refreshable and time efficient but it costs eight thousand US dollars at least a piece, which is very expensive [16].

The latest development that ensures the user adequate reading and a time efficient process while reading a text is the Rotating Text to Braille converter. This development is not yet commercialized. It makes a continuous reading for a text easier as the Braille characters are centered on a circled plate that rotates while the user has his or her fingers on the same position throughout the whole process. The circled plate rotates at a speed controlled by the user. The device simply translates the text that is on a computer screen into Braille representations and outputs them continuously on the rotating wheel. A main advantage of this device is that it costs a slightly higher than two thousand dollars, which is cheaper than the previous Braille devices. However, users are not able to review what they have already read since the wheel is continuously rotating in one direction. Moreover, it was not very practical for users, since many of them complained that it was hard to read and skim texts easily.

Current studies in the area of the development of Braille display devices are addressing the two major problems found in the existent devices. The first one is the high cost of the available devices: a simple output device costs thousands of dollars. The other major problem is the small number of characters that can be displayed by the Braille Output Devices [17].

A new concept is being developed by researchers at the North Carolina State University, NC, USA. This concept is called "hydraulic and latching mechanism". This mechanism uses Electroactive Polymers as actuators. The dots can be thought of as tiny containers filled with a liquid. When exposed to electricity, the sides will bend and push the liquid upward, creating a small bulge at the top. After raising the dot, a latching mechanism is used 
to maintain the current position of the dot and support the weight being applied by the fingers of the person reading the character. The polymers used in this mechanism are cheap. This solves the price problem of the device. This mechanism also allows the display of full pages with refreshable output. As a result, the user is able to scan a whole page while looking for specific information. He or she will also take full advantage of the computer applications. This mechanism also allows the representation of images [18].

The "hydraulic and latching" mechanism is still under development. Experiments are still being done to prove the validity of this concept. Though researchers presented their discoveries about the hydraulic part of the concept, proving the feasibility of this technology; however, the latching mechanism still needs a proof-of-concept model. Developing the "hydraulic and latching" concept will solve all the problems associated with the Braille display devices. First, the Electroactive Polymers that are used as actuators are both strong and inexpensive; thus, solving the cost issue. Second, a full page can be displayed using this mechanism. This solves the problem of the low number of characters that can be displayed simultaneously. Third, this mechanism can also solve the problem of graphical representations. It allows the translation of images into tactile displays. The images are represented as raised dots, each representing a pixel [18].

\section{Materials and Methods}

The design of the Multi-Lingual Braille System Output Device with Audio Enhancement includes two parts: the hardware device and the software program. In this section, the materials used in the system as well as the methods are detailed. The materials used in the implementation of the Multi-Lingual Braille System Output Device with Audio Enhancement are: 1) Robust PiezoActuated 8-Dot Braille Cell, 2) HV507, 3) PIC 16F877A Microcontroller, 4) C\# Programming Language, 5) Dual Power Supply, 6) Control Switches, 7) Windows Operating System, and 8) Universal Serial Bus Communication Channel.

\subsection{Robust Piezo-Actuated 8-Dot Braille Cell (Metec AG, Stuttgart, Germany)}

The Robust Piezo-Actuated 8-dot Braille Cell (Figure 1), also known as Braille Cell P16, is a device that receives the character to be outputted as electric signal, and simultaneously outputs the Braille character according to the inputted signal [19].

\subsection{HV507 (Supertex Inc., CA, USA)}

HV507 is a low voltage serial to high voltage parallel

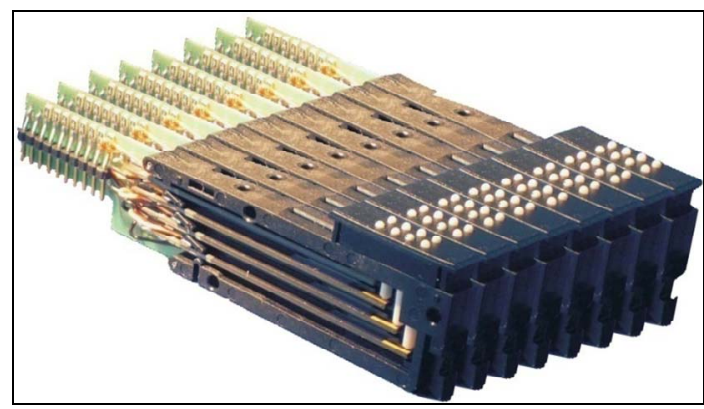

Figure 1. The P16 Braille cells (metec AG, Stuttgart, Germany).

converter with 64 high voltage push-pull outputs. It is used to convert the TTL voltage provided by the microcontroller into high voltages capable of actuating the Braille dots [20].

\subsection{PIC 16F877A Microcontroller}

The microcontroller is the component responsible for all the computations that are required by the device. The microcontroller receives the data from the input module, performs computations, and sends pulses to control the output.

\subsection{C\# Programming Language (Microsoft Corp., Redmond, WA, USA)}

$\mathrm{C \#}$ is the programming language used to write the code responsible for the interaction between the computer and the output device via the USB port of the computer.

\subsection{Dual Power Supply}

The dual power supply is used to convert the main voltages (220 V AC) into $5 \mathrm{~V}$ and $200 \mathrm{~V}$ DC voltages required for the operation of the microcontroller and the Braille Cells respectively. The power supplies are composed of one step-up and two step-down transformers, followed by a rectifier, and a filter.

\subsection{Control Switches}

The device is controlled by several control switches. The control switches are "on" and "off" as well as the arrow buttons that control which characters are to be displayed. Moreover, a sound button is added to induce the pronouncing of the outputted characters. In addition, a reset switch is used to restart the whole device.

\subsection{Windows Operating System (Microsoft Corp., Redmond, WA, USA)}

A personal computer or laptop running Windows Operating System is needed. The computer used for testing the project has the following specifications: Windows Vista, 2.3 GHz Processor Speed, 3 GB RAM, 250 GB 


\section{Hard Disk Drive.}

\subsection{Universal Serial Bus Communication Channel}

The Universal Serial Bus (USB) cable connects the personal computer to the Braille Output Device. The USB cable is responsible for the transmission of the data from the personal computer Universal Serial Bus port to the main Microcontroller.

The device is built and packed into a case, conforming to the international electrical safety measures [21]. The methods used to implement the project are divided into a software part and a hardware part.

\subsubsection{Software}

This project specifically helps the user to read a text from a file, or process a dynamically given data from a keyboard. The software is written using the $\mathrm{C \#}$ programming language. The application installed on the computer opens the needed file and reads the data. After the text is entered, the program converts each character into its equivalent binary code, indicating a specific Braille character. Each eight processed codes are sent via a USB port to the device.

The program (Figure 2) starts three simultaneously running threads. The first thread is responsible for the graphical user interface (GUI). The user has the option to choose between reading from a file and reading dynamically. The data will then be read from a chosen text file or from the onscreen textbox, converted into its precoded binary representation and sent via the USB port. In addition, the user has the ability to select the type of text to be sent. For instance, he can choose the 8-dot, the

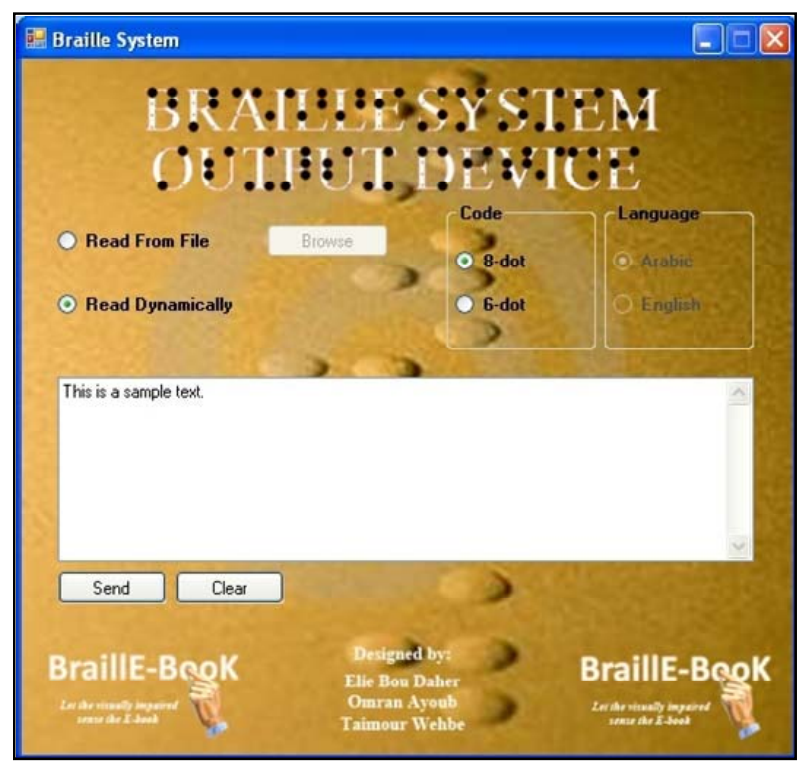

Figure 2. The Braille system output device software GUI.
English 6-dot, or the Arabic 6-dot representation. Moreover, this program is supported by a sound feature which helps the user in navigating through the system's graphical interface. The second thread waits for any intervention from the user via the control buttons. When a button is pressed, the program receives its corresponding code to perform the required action. The third thread is responsible for the pronunciation of the displayed characters. When the sound button is pressed, the program runs this thread to play the corresponding sounds.

The data received from the USB port is processed by a 16F877A Microcontroller. When the data is received, each number is stored in memory and then sent serially, at each clock edge, to the Braille cells. The microcontroller keeps checking and waiting for any intervention from the user via the control buttons so as to issue an order for the computer program, indicating the button pressed.

\subsubsection{Hardware}

Figure 3 depicts the block diagram of the hardware with the interaction of all units. The serially sent data is fed to a shift register. This shift register multiplexes the data and changes the serial data to 64 multiplexed channels. Each eight channels are directed to one Braille cell, and each channel is directed to a specific pin. The TTL voltages issued from the shift register and the microcontroller activate the high DC voltage $(200 \mathrm{~V})$ in order to raise or lower the respective pins. So, when a specific channel is given a high level $(5 \mathrm{~V})$, the $200 \mathrm{~V}$ fed to the Braille cell forces the corresponding pin to be raised. On the other hand, when the channel is given a low level $(0 \mathrm{~V})$, the $200 \mathrm{~V}$ fed to the Braille cell forces the corresponding pin to be lowered.

Moreover, the user is provided with four control buttons (Figure 4). The first button allows him to display the next eight characters in the text. The second button displays the previous characters, enabling the user to review the previous sentences. A third button allows the user to jump to the second paragraph, allowing the user to scan through a text faster. The fourth button does the opposite operation. Other buttons that perform some other specific operations can also be incorporated into the design.

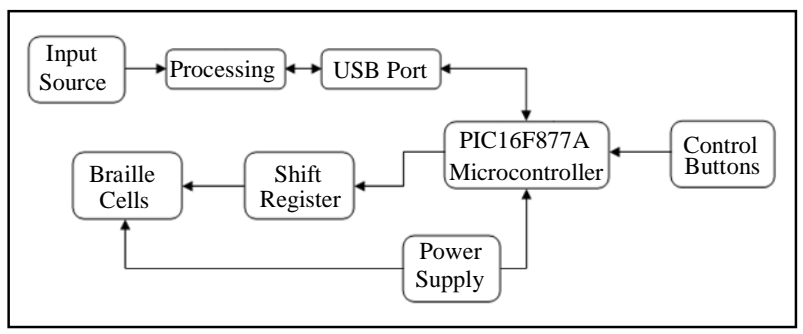

Figure 3. The block diagram of the device. 


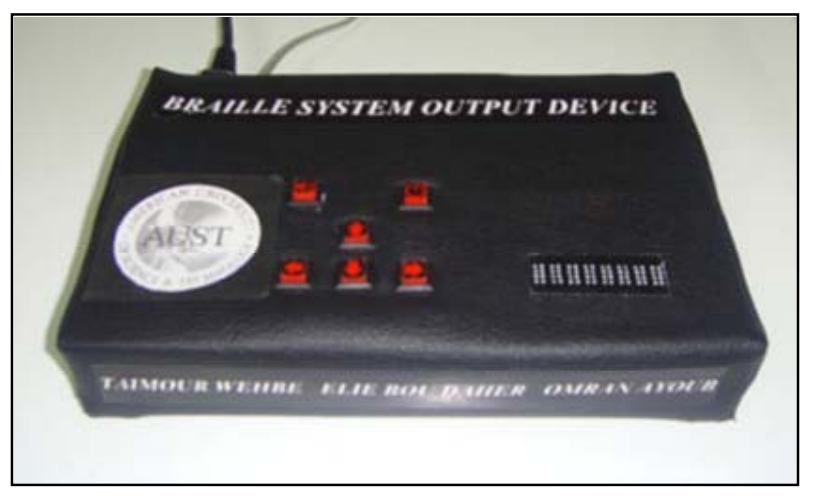

Figure 4. The multi-lingual Braille system output device with audio enhancement developed in this study.

The power supply used to power up the device is a dual power supply that takes $220 \mathrm{~V}$ AC as input. Three transformers are used to step down the high voltage into three different voltages. One transformer is used to create the needed $5 \mathrm{~V}$, while the other two are used to create the needed $200 \mathrm{~V}$. All voltages are rectified, filtered and regulated.

\section{Results and Discussion}

The testing of the project was divided into two phases. First, the different project parts were tested separately. Then, an overall testing was done to confirm the functionality of the process as a whole.

The initial testing of the different parts of the project was divided into several steps. Starting with the software part, the conversion of the characters to their corresponding Braille binary representation was validated using the command line output. The program was given a list of all possible characters as an input to the command line, and then the generated output was compared to that of the Braille representation of these characters. Each character was assigned a specific number. The program took the text and divided it into characters. Each character was then converted to its corresponding number. Then, the program displayed the converted numbers on the command line. These numbers were then compared to that of the desired ones in order to ensure that the conversion was correct.

The next step was testing the functionality of the control buttons. The control buttons were simulated in the program. When a button was pressed, the change in the output of the program was compared to that of the desired one: The right button displayed the next eight characters in the output; while the left button displayed the previous ones. The down button jumped to the next paragraph, and the up button jumped to the previous one. The sound button initiated the audio feature of the program.

Testing the transmission of data between the computer and the microcontroller was done afterwards. This step was divided into two sub-steps. The first one was sending data from the computer to the microcontroller. The second one was sending data from the microcontroller back to the computer. During each sub-step, specific numbers were generated and then sent through the transmission line. The obtained numbers were then compared to that of the generated ones.

The hardware testing was the final step. Each hardware part was tested separately. The voltages generated by the power supplies were measured and compared to that of the desired ones. The robustness of the supplies was tested against loading effects. In addition, the Braille cells were tested separately. Test numbers were generated by the microcontroller and then fed to the Braille cells. A combination of raised dots was formed. The displayed representations were then compared with the actual Braille representations of those numbers.

The next phase aimed to test end-to-end functionality. This phase was done by giving a test input file to the program and checking the generated output. In addition, each control switch was tested by viewing the change in the output against its desired function.

All 8-dot characters, 6-dot English characters and 6dot Arabic characters were given as test inputs. The results obtained proved the validity of the design and of the implementation. The actual output (Figures 5-7) conformed to the desired one with a hundred percent accuracy. In addition, the control buttons were able to achieve their respective purposes without errors. Moreover, the simplification of the hardware design reduced the high cost of the device. Furthermore, the device proved to be power efficient, having a maximum power consumption of $1 \mathrm{~W}$.

After having ensured that the project has accomplished a hundred percent functionality, the device is now undergoing a series of tests at several institutes for the Blind by Braille-qualified teachers and blind students. An average of the percentage of correctly read characters is to be computed. Moreover, any suggestions provided by these experts will be taken into consideration for future plans.

\section{Conclusions and Future Work}

The Blind and the visually impaired individuals in Lebanon make up an important part of the Lebanese community. These individuals still suffer from setbacks concerning reading and communicating through computers. In addition, the market has shown minor concern towards such individuals. Due to this fact, and in order to help the blind and the visually impaired individuals read and communicate, the Multi-Lingual Braille System Output Device with Audio Enhancement is introduced in the 


\begin{tabular}{|c|c|c|}
\hline Characters & Braille Representations & Output \\
\hline A B C D E F G H & 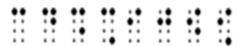 & 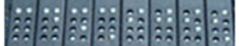 \\
\hline I J K L M N O P & 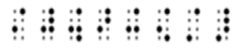 & \\
\hline QR S T U VWX & לִ: & \\
\hline $\mathrm{YZ}$ & 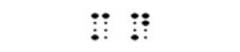 & \\
\hline$a b c d e f g h$ & ט: & \\
\hline $\mathrm{ijk} 1 \mathrm{mnop}$ & לִ: & \\
\hline qrstuvwx & : & $10 \hat{\theta}=\hat{0}$ \\
\hline $\mathrm{yz}$ & 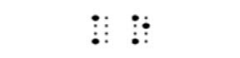 & \\
\hline 01234567 & 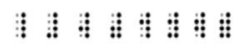 & \\
\hline 89 & : & \\
\hline
\end{tabular}

Figure 5. 8-dot English characters results.

\begin{tabular}{|c|c|c|}
\hline Characters & Braille Representations & Output \\
\hline$a b c d e f g h$ & 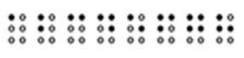 & $\therefore: \therefore:$ \\
\hline$i j k 1 m n o p$ & 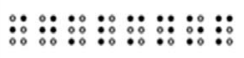 & \\
\hline qrstuvw & 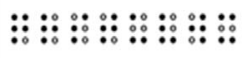 & 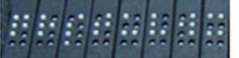 \\
\hline y z & $:::$ & \\
\hline 0123 & • : & 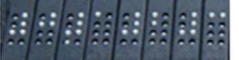 \\
\hline 4567 & 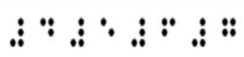 & $\therefore: \because \vdots$ \\
\hline 89 & : : $: \quad \therefore$ & in: \\
\hline
\end{tabular}

Figure 6. 6-dot English characters results.

\begin{tabular}{|c|c|c|}
\hline Characters & Braille Representations & Output \\
\hline ا & $\cdot:::^{\prime}::^{\prime}: \because \cdot:$ & $\because: \because \ddot{0}:$ \\
\hline 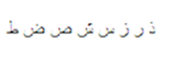 & :: ::": : : : : : : & $\therefore \therefore \therefore \therefore: \therefore: \vdots 0$ \\
\hline 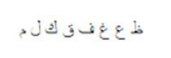 & 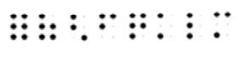 & 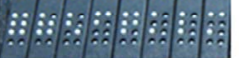 \\
\hline 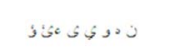 & 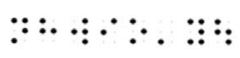 & 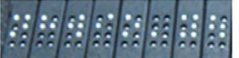 \\
\hline $3 ! i$ & $\therefore:$ & $\therefore$ \\
\hline
\end{tabular}

Figure 7. 8-dot Arabic characters results.

hope of solving this problem, and in satisfying the needs of the blind community.

This device satisfies the needs of the visually impaired individuals in Lebanon and the Arab World since it enables the translation of both English and Arabic text into their Braille representations. Furthermore, the software associated with the device has a sound feature that will help the blind access the program and control its output. One of the major advantages of the device is its relatively low cost in comparison to similar devices. In addition, several buttons are added to help the user skim through the text he or she is reading.

The implementation of the device was divided into several stages. The first stage was the software development stage. It included the development of the microcontroller software and the C\# program. The second stage was the hardware implementation phase, which included the design and assembly of the whole device. After the hardware part of the project was completed, the software and the hardware were tested separately; the acquired results were in accordance to the expected outcomes. The final stage consisted of an overall testing. In this stage, the whole process was tested and operated without any error.

Unquestionably, the development of this Multi-Lingual Braille System Output Device with Audio Enhancement is the initial step for building and commercializing Braille devices in Lebanon and the Arab World. The continuous development of such devices is essential for the visually impaired to keep up with the technological advancements. Nevertheless, such a device can still undergo many changes and improvements. For instance, the number of displayed characters in this prototype can be increased to build a more practical device. In addition, many features can be added to the device to make it fit to satisfy the users' needs, such as adding new languages, introducing Grade 2 Braille System, and providing the capability of printing using Braille embossers. Moreover, an input feature can be added to the device to help blind individuals input instructions to the computer, using the Braille language.

In the light of helping the blind communicate with the outside world, some software improvements can also be integrated. For example, a website can be hosted online in order to enable the blind person to chat, send, and receive e-mails using the Braille language. An online dynamic translation and communication can occur with the chat program and the Multi-Lingual Braille System Output Device with Audio Enhancement simultaneously. As a result, the blind individual will be able to interact with any person worldwide in real time.

\section{Acknowledgments}

This work was supported by funds from the Research Council of the American University of Science \& Technology (AUST, Beirut, Lebanon).

The authors thank Dr. Ziad O. Abu-Faraj, Chairperson, Department of Computer and Communications Engi- 
neering at AUST for his guidance and Mrs. Henriette Skaff in the Department of Languages and Translation at AUST for her invaluable help in editing this article.

\section{REFERENCES}

[1] C. Marsan and C. Coudert, "Louis Braille-A Brief Overview," Proceedings of the International Conference Braille 1809-2009: Writing with Six Dots and Its Future, Paris, 5-8 January 2009.

[2] M. Donaldson and T. Butler, "Louis Braille (History Maker Bios)," Lerner Publishing Company, Minneapolis, 2007.

[3] P. Ament, "Fascinating Facts about the Invention of Braille by Louis Braille in 1824,” Idea Finder, 2006. http://www.ideafinder.com/history/inventions/braille.htm

[4] P. Kimbrough, "How Braille Began,” Enabling Technologies, 2008. http://www.brailler.com/braillehx.htm

[5] Wikipedia, "Braille,” Wikipedia: The Free Encyclopedia, Wikimedia Foundation Inc., 2011. http://en.wikipedia.org/wiki/Braille

[6] “World Braille Usage,” Library of Congress, Washington DC, 1990, p. 132.

[7] “Braille Codes,” Tiresias, 2009. http://www.tiresias.org/research/guidelines/braille_codes/ index.htm

[8] G. Kapperman and J. Sticken, "Using the Braille Lite to Study Foreign Languages,” Journal of Visual Impairment \& Blidness, Vol. 97, No. 11, 2003, pp. 1-13.

[9] “Hardware Overview: Braille Lite," Indiana University, 2008. http://www.indiana.edu/ iuadapts/technology/hardware/b raille/braille_lite.html

[10] "Braille Lite Millennium Series M20," Dancing Dots, 2007. http://www.dancingdots.com/prodesc/m20notetaker.htm
[11] L. Garber, "Blind, Deaf Engineer Develops Computerized Braille Machine,” Computer, Vol. 35, No. 12, 2002, 27 p.

[12] N. Efron, “Optacon-A Replacement for Braille?” The Australian Journal of Optometry, Vol. 60, No. 118, 1977, pp. 118-128.

[13] J. Halliday, “A Fresh Look at Braille, Closing the Gap,” Kurzweil Educational Systems, 2003-2004. http://www.kurzweiledu.com/files/Dec\%20Jan\%20Fresh \%20Look.pdf

[14] Synapseadaptive, “Alva Braille Display 570 Satellite Pro,” 2002.

http://www.synapseadaptive.com/alva/alva_pro/alva_570 _satellite_pro.htm

[15] Indiana University, "Hardware Overview: Alva Refreshable Braille,” 2006.

http://www.indiana.edu/ iuadapts/technology/hardware/b

[16] J.-M. Chung, K. Ramasamy, V. Kotikalapudi, Z. Mulla, G. Thiyagarajan, M. Weiser, G. Scheets and R. Sharda, "Virtual Laboratory Education for Persons with Vision Disabilities," The 2002 Midwest Symposium on Circuits and Systems, Tulsa, 4-7 August 2002, pp. II-617-II-620.

[17] J. Briant, “Louis Braille, Teacher of the Blind,” Chelsea House Publishers, New York, 1994, p. 111.

[18] N. Di Spigna, P. Chakraborti, D. Winick, P. Yang, T. Ghosh and P. Franzon, "The Integration of Novel EAPBased Braille Cells for Use in a Refreshable Tactile Display," Proceedings of the 12th International Conference on Electroactive Polymer Actuators and Devices, San Diego, March 2010, p. 9.

[19] Metec AG, “Module P16 Datasheet," Stuttgart, Germany, 2010. http://www.metec-ag.de/P16.pdf

[20] Supertex Inc., "HV507 Datasheet,” 2008. http://www.supertex.com/pdf/datasheets/HV507.pdf

[21] Underwriters Laboratories, "Scopes for UL Standards," 2011. http://ulstandardsinfonet.ul.com/scopes/ 